\title{
The origin of the hard X-ray tail in neutron-star X-ray binaries
}

\author{
P. Reig ${ }^{1,2}$ and N. Kylafis ${ }^{2,1}$ \\ 1 IESL, Foundation for Research and Technology-Hellas, 71110 Heraklion, Greece \\ e-mail: pau@physics.uoc.gr \\ 2 Physics Department \& Institute of Theoretical \& Computational Physics, University of Crete, 70013 Heraklion, Greece \\ e-mail: kylafis@physics.uoc.gr
}

Received 11 February 2016/ Accepted 28 April 2016

\begin{abstract}
Context. Neutron star X-ray binaries emit a compact, optically thick, relativistic radio jet during low-luminosity, usually hard states, as Galactic black-hole X-ray binaries do. When radio emission is bright, a hard power-law tail without evidence for an exponential cutoff is observed in most systems.

Aims. We have developed a jet model that explains many spectral and timing properties of black-hole binaries in the states where a jet is present. Our goal is to investigate whether our jet model can reproduce the hard tail, with the correct range of photon index and the absence of a high-energy cutoff, in neutron-star X-ray binaries.

Methods. We performed Monte Carlo simulations of the Compton upscattering of soft, accretion-disk or boundary layer photons in the jet and computed the emergent energy spectra, as well as the time lag of hard photons with respect to softer ones as a function of Fourier frequency. We fit the energy spectra with a power law modified by an exponential cutoff at high energy.

Results. We demonstrate that our jet model naturally explains the observed power-law distribution with photon index in the range $1.8-3$. With an appropriate choice of the parameters, the cutoff expected from Comptonization is shifted to energies above $\sim 300 \mathrm{keV}$, producing a pure power law without any evidence for a rollover, in agreement with the observations.

Conclusions. Our results reinforce the idea that the link between the outflow (jet) and inflow (disk) in X-ray binaries does not depend on the nature of the compact object, but on the process of accretion. Furthermore, we address the differences between jets in black-hole and neutron-star X-ray binaries and predict that the break frequency in the spectral energy distribution of neutron-star X-ray binaries, as a class, will be lower than that of black-hole binaries.
\end{abstract}

Key words. stars: neutron - binaries: close - X-rays: binaries

\section{Introduction}

In black-hole (BHXB) and neutron-star (NSXB) X-ray binaries, the transfer of mass from the optical companion to the compact star is mediated by an optically thick, geometrically thin, accretion disk. Because the mass-to-radius ratio in neutron stars and black holes is similar ${ }^{1}$, the characteristic velocities and dynamical time scales near the compact object are also similar $(v \sim 0.5 c$, $t \lesssim 1 \mathrm{~ms}$ ). Thus, it is not surprising that BHXB and NSXB show very similar X-ray spectral and temporal characteristics (van der Klis 1994, 2005; Maccarone \& Coppi 2003; Barret 2004; Di Salvo et al. 2006; Muñoz-Darias et al. 2014).

It is generally accepted that the X-ray spectral continuum of BHXB and NSXB results from the sum of two main components: one thermal component which follows a blackbody distribution and another non-thermal component that is best described as a power law. The thermal component dominates at lower energies, hence it is referred to as the soft component, while the power-law component extends up to a few hundred $\mathrm{keV}$ and it is often known as the "hard tail" (Barret 2004; McClintock \& Remillard 2006; Lin et al. 2007; Done et al. 2007; Church et al. 2012).

In $\mathrm{BHXB}$, the soft thermal component is attributed to the emission from the innermost, hotter regions of the accretion disk, while the hard power law results from Comptonization of lowenergy photons by energetic electrons. The physical origin of the

\footnotetext{
A $5 M_{\odot}$ black hole has a Schwarzchild radius of $\sim 15 \mathrm{~km}$.
}

Comptonizing medium could be an advection-dominated accretion flow (Narayan \& Yi 1994; Esin et al. 1997), a low angular momentum accretion flow (Ghosh et al. 2011; Garain et al. 2012), or a radio jet (Band \& Grindlay 1986; Georganopoulos et al. 2002; Reig et al. 2003; Giannios 2005; Markoff et al. 2005).

In NSXB, the emission spectrum is made more complex by the presence of a hard surface. Thus below $\sim 10 \mathrm{keV}$, NSXB may display two soft components: a single-temperature blackbody from the neutron star surface or from the boundary layer between the disk and the neutron star and a multi-temperature blackbody from the accretion disk (Mitsuda et al. 1984; Gilfanov et al. 2003). The temperature of the neutron star photons is expected to be higher $(k T \sim 2-3 \mathrm{keV})$ than that of the disk soft photons $(k T \sim 1 \mathrm{keV})$, because the effective area of the neutron star surface is more compact than that of the accretion disk (Farinelli et al. 2007). Above $\sim 20 \mathrm{keV}$, the most prominent spectral feature in the X-ray spectrum of NSXB is a power-law distribution (the hard tail), that usually extends up to $200-300 \mathrm{keV}$ without evidence of a cutoff (Di Salvo et al. 2000, 2001; Iaria et al. 2001; D'Amico et al. 2001; Farinelli et al. 2005; Migliari et al. 2007; Ding et al. 2011).

Both kinds of binary systems exhibit distinct spectral states in response to changes in the mass accretion rate, for example in the course of an outburst. The main states are generally referred to as soft and hard, depending on whether the bulk of the luminosity is radiated below or above a few $\mathrm{keV}$, respectively. 
Another property common to both BHXB and NSXB is the presence of radio jets (Fender et al. 2004; Migliari \& Fender 2006). Optically thick, compact, steady radio emission is detected during the low (in the $1-20 \mathrm{keV}$ band) X-ray lumonisity hard state, while optically thin radio flares occur during transitions from the hard to the soft sate. The jet is quenched in the soft state, when the X-ray luminosity approaches the Eddington limit (Paizis et al. 2006; Migliari et al. 2007; Miller-Jones et al. 2010).

A useful way to describe the rich phenomenology exhibited by these systems in the X-ray band is the hardness-luminosity diagram (HLD). In the HLD, BHXB and NSXB display distinct curves, along which the sources move smoothly as the mass accretion rate varies. In Kylafis \& Belloni (2015), a physical inerpretation was offered for the phenomenology of BHXB along the $q$-shaped curve in the HLD. In NSXB, the shape of the curve in the HLD devides them into $Z$ sources and atoll sources (Hasinger \& van der Klis 1989; van der Klis 2006). The three branches that form the Z-shaped HLD are called horizontal (HB), normal (NB), and flaring (FB) branches. The HB corresponds to the hardest X-ray state, while the FB is the soft state. In the case of atoll NSXB, the branches are called island and banana branches, with subcategories such as extreme island and lower and upper banana. The extreme island branch corresponds to the hardest spectral sate. The hard tail has been detected in almost all the currently known $Z$ sources (Di Salvo et al. 2002) and many of the atoll sources (Paizis et al. 2006).

The association of the compact radio jet and the X-ray hard tail with a specific region of the source in the HLD is well documented in BHXB (Fender et al. 2009) and NSXB (Paizis et al. 2006; Migliari \& Fender 2006). Both the radio emission and the strength of the hard tail become weaker at higher accretion rates. Radio and hard X-rays show the strongest intensity in the hard states of BHXB, the horizontal branch of Z-NSXB (Hjellming et al. 1990; Migliari et al. 2007; D'Aí et al. 2007), and the island or lower banana states in atoll-NSXB (Migliari et al. 2003). In $\mathrm{BHXB}$, radio emission is absent in the soft sate. In some NSXB, radio emission may be still detected in the softer states, albeit highly reduced (Homan et al. 2004).

While there is a general consensus that the radio emission is produced by a compact jet, the physical origin of the hard tail is not well understood. It naturally arises from Comptonization, however, the details of how the electrons acquire their energy (thermal, non-thermal, or bulk-motion) and the source of seed photons (neutron-star surface, inner accretion disk, synchrotron photons) remain unclear (Barret et al. 2000; Di Salvo et al. 2006; Paizis et al. 2006; Lin et al. 2007; Markoff et al. 2005; Farinelli et al. 2009; Revnivtsev et al. 2014). A weak hard tail contributing a small percentage of the total flux $(\$ 3 \%)$ is also detected in the soft state, when the jet is quenched or absent (see, e.g., McClintock \& Remillard 2006, and references therein). In this state, the power-law tail is thought to come from Comptonization in non-thermal flares above and below the thermal disk (Poutanen et al. 1997; Gierliński et al. 1999).

In a series of papers (Reig et al. 2003; Giannios et al. 2004; Giannios 2005; Kylafis et al. 2008; Reig \& Kylafis 2015), we showed that Compton upscattering of soft photons from the accretion disk in the jet can explain a number of observational relations between the spectral and timing parameters in the hard state of BHXB. Our results clearly demonstrate that jets play a central role in all the observed phenomena, not only in the radio emission. Motivated by the similarities of the X-ray spectral continuum in BHXB and NSXB and especially by the clear connection of the presence of a hard tail with a radio-loud state of the source, we investigate whether our model can also account for the spectral properties of NSXB.

Our objective in this paper is to demonstrate that emission from the neutron-star surface plus Comptonization in a jet can reproduce the observed spectra of bright NSXB, namely a soft thermal component that is well described by a blackbody distribution with $k T=2-3 \mathrm{keV}$ and a hard tail that follows a powerlaw with photon index in the range 1.8-3 with no evidence for a cutoff up to $200-300 \mathrm{keV}$.

\section{The model}

The model that we have used in this work is identical to that used in Reig \& Kylafis (2015). For our Monte Carlo code we follow Pozdnyakov et al. (1983). Photons from a blackbody distribution of temperature $k T_{\mathrm{bb}}$ are injected at the base of the jet with an upward isotropic distribution. As the photons travel through the medium, they experience Compton scatterings with the spiraling electrons. In each scattering, the photons on average gain energy from the bulk motion (i.e., $v_{\|}$and $v_{\perp}$ ) of the electrons. Comptonization can occur everywhere in the jet. The optical depth to electron scattering, the energy change and the new direction of the photons after scattering are computed using the corresponding relativistic expressions. Each model is run for $10^{7}$ photons. More details of how the code works can be found in Kylafis et al. (2008).

Since we are not interested in reproducing the radio spectrum (see Giannios 2005), we have assumed mono-energetic electrons in the jet with Lorentz factor equal to the smallest in the distribution, namely

$\gamma_{\min }=\frac{1}{\sqrt{1-\left(v_{\|}^{2}+v_{\perp}^{2}\right) / c^{2}}}$,

where $v_{\|}=v_{0}=$ const. is the terminal velocity of the jet and $v_{\perp}$ is the smallest peprendicular velocity of the electrons in the lab frame.

The flow velocity in the jet is given by

$v_{\|}(z)= \begin{cases}v_{0}\left(z / z_{1}\right)^{p} & \text { if } 0<z \leqslant z_{1} \\ v_{0} & \text { if } z>z_{1},\end{cases}$

where $z_{1}$ and $p$ are parameters. In other words, the jet has an acceleration region of thickness $z_{1}$, beyond which the flow has constant velocity $v_{0}$.

For a parabolic jet, i.e., one whose radius at height $z$ is $R(z)=$ $R_{0}\left(z / z_{0}\right)^{1 / 2}$, the electron density is obtained from the continuity equation and for $z>z_{1}$ it is inversely proportional to $z$.

The fixed parameters of our models and their reference values are: the radius $R_{0}=50 R_{\mathrm{NS}}$ of the base of the jet, where $R_{\mathrm{NS}}=1.25 \times 10^{6} \mathrm{~cm}$ is the radius of the neutron star, the distance $z_{0}=1 R_{\mathrm{NS}}$ of the bottom of the jet from the neutron star center, the height $H=10^{5} R_{\mathrm{NS}}$ of the jet, the terminal velocity $v_{0}=0.8 c$ of the jet, the thickness $z_{1}=5 R_{\mathrm{NS}}$ of the acceleration zone, the exponent $p=1 / 2$, and the temperature $k T_{\mathrm{bb}}=2.5 \mathrm{keV}$ of the soft-photon input.

In our model, the parameters that we have varied are the Thomson optical depth $\tau_{\|}$along the axis of the jet and the minimum Lorentz factor $\gamma_{\min }$. Since $v_{0}$ is a constant in our models, the variation of $\gamma_{\min }$ is equivalent to a variation in $v_{\perp}$.

Because the jet is relativistic, the results also depend on the angle $\theta$ of observation with respect to the jet axis. Here we consider an intermediate range of observing angles $0.2<\cos \theta<$ 0.6. Practically, for the Monte Carlo simulation this means that we count only photons that leave the jet in this range of angles. 


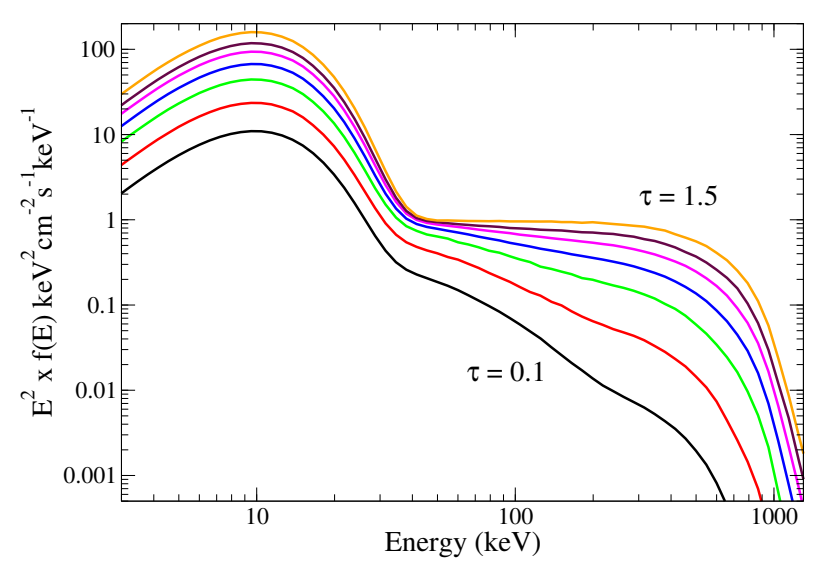

Fig. 1. Emergent spectra from the Monte Carlo simulations. The models plotted correspond to a fixed $\gamma_{\min }=3.34\left(v_{\perp}=0.52 c\right)$ and optical depth $\tau=0.1,0.25,0.5,0.75,1.0,1.25,1.5$. The input source of photons follows a blackbody distribution with $k T=2.5 \mathrm{keV}$. The normalization of the input blackbody was chosen so that the hard component contributes about $5 \%$ of the $0.1-300 \mathrm{keV}$ luminosity. In the energy range covered by current missions $(<300 \mathrm{keV})$ the hard tail shows no break.
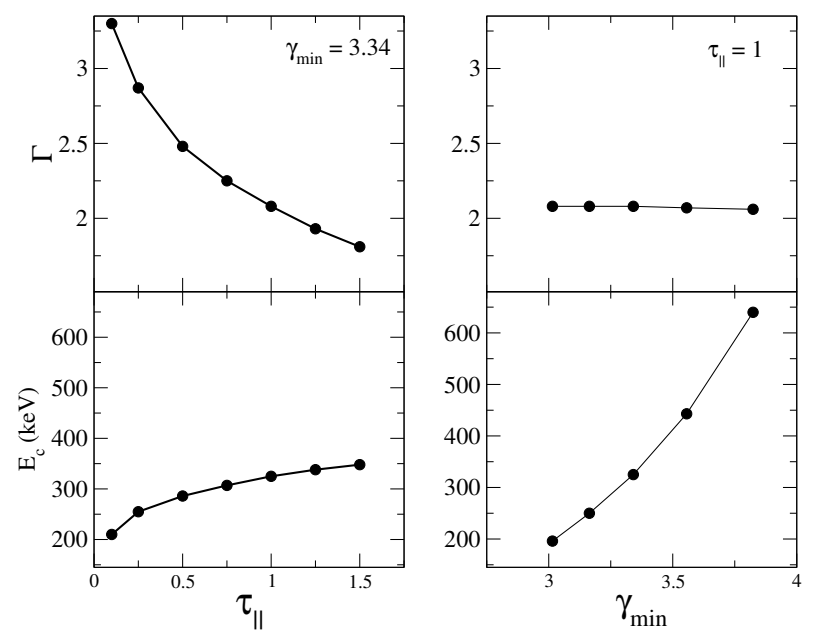

Fig. 2. Photon index and cutoff energy as functions of $\tau_{\|}$and $\gamma_{\min }$. In the left-hand panels, each point represents a calculation with the same $\gamma_{\min }=3.34\left(v_{\perp}=0.52 c\right)$ and different $\tau_{\|}$. In the right-hand panels, each point represents a calculation with the same $\tau_{\|}=1$ and different $\gamma_{\min }$. The scale of the $Y$-axis was left the same to faciliate the comparison.

\section{Results}

The spectral parameters relevant to the present study are the photon index of the hard-tail component and the cutoff energy. We have investigated the dependence of these two quantities on the choice of two parameters of our model, namely the optical depth and the electron velocity (see Table 1 ).

Figure 1 shows the emerging spectra that result from our simulations using the reference values of the parameters reported in Sect. 2 with $\gamma_{\min }=3.34\left(v_{\perp}=0.52 c\right)$ and various optical depths $\tau_{\|}=0.1,0.25,0.5,0.75,1.0,1.25$, and 1.5 . As seen in the figure, although the spectra roll over above a certain energy, this energy is well above $200-300 \mathrm{keV}$. Thus, up to $200-300 \mathrm{keV}$, the spectra are essentially pure power-laws with photon index between $1.8-3$, in agreement with the observations. Figure 2 shows the photon index $\Gamma$ and the cutoff energy $E_{\mathrm{c}}$ as functions of optical depth $\tau_{\|}$and $\gamma_{\min }$ (or equivalently $v_{\perp}$ ). The scale of the vertical axis was kept the same in the two plots to better assess the differences. The cutoff energy has a weak dependence on $\tau_{\|}$for the
Table 1. Monte Carlo simulation results.

\begin{tabular}{cccc}
\hline \hline$\tau_{\|}$ & $\gamma_{\min } / v_{\perp}(c)$ & $\Gamma$ & $E_{\mathrm{c}}(\mathrm{keV})$ \\
\hline 0.10 & $3.015 / 0.50$ & 3.30 & 133 \\
0.10 & $3.164 / 0.51$ & 3.31 & 172 \\
0.10 & $3.341 / 0.52$ & 3.28 & 223 \\
0.10 & $3.556 / 0.53$ & 3.26 & 314 \\
0.10 & $3.824 / 0.54$ & 3.27 & 571 \\
0.25 & $3.015 / 0.50$ & 2.82 & 151 \\
0.25 & $3.164 / 0.51$ & 2.82 & 195 \\
0.25 & $3.341 / 0.52$ & 2.81 & 253 \\
0.25 & $3.556 / 0.53$ & 2.79 & 355 \\
0.25 & $3.824 / 0.54$ & 2.79 & 566 \\
0.50 & $3.015 / 0.50$ & 2.44 & 173 \\
0.50 & $3.164 / 0.51$ & 2.44 & 219 \\
0.50 & $3.341 / 0.52$ & 2.43 & 288 \\
0.50 & $3.556 / 0.53$ & 2.42 & 392 \\
0.50 & $3.824 / 0.54$ & 2.41 & 588 \\
0.75 & $3.015 / 0.50$ & 2.21 & 187 \\
0.75 & $3.164 / 0.51$ & 2.20 & 238 \\
0.75 & $3.341 / 0.52$ & 2.20 & 309 \\
0.75 & $3.556 / 0.53$ & 2.19 & 422 \\
0.75 & $3.824 / 0.54$ & 2.18 & 604 \\
1.00 & $3.015 / 0.50$ & 2.01 & 195 \\
1.00 & $3.164 / 0.51$ & 2.02 & 251 \\
1.00 & $3.341 / 0.52$ & 2.01 & 322 \\
1.00 & $3.556 / 0.53$ & 2.01 & 438 \\
1.00 & $3.824 / 0.54$ & 2.00 & 628 \\
1.25 & $3.015 / 0.50$ & 1.88 & 205 \\
1.25 & $3.164 / 0.51$ & 1.89 & 262 \\
1.25 & $3.341 / 0.52$ & 1.89 & 344 \\
1.25 & $3.556 / 0.53$ & 1.88 & 461 \\
1.25 & $3.824 / 0.54$ & 1.88 & 665 \\
1.50 & $3.015 / 0.50$ & 1.73 & 210 \\
1.50 & $3.164 / 0.51$ & 1.74 & 268 \\
1.50 & $3.341 / 0.52$ & 1.74 & 347 \\
1.50 & $3.556 / 0.53$ & 1.73 & 457 \\
1.50 & $3.824 / 0.54$ & 1.73 & 640 \\
\hline & & &
\end{tabular}

Notes. Different models correspond to different values of the optical depth $\left(\tau_{\|}\right)$and perpendicular component of the electron velocity $\left(\gamma_{\min }\right)$. All the remaining parameters of the model were fixed at the reference values (see Sect. 2).

range of optical depths considered, but it is strongly dependent of $\gamma_{\min }$. This is expected because the cutoff is mainly determined by the energetics of the electrons (Giannios 2005). In contrast, the photon index strongly depends on $\tau_{\|}$, but only weakly on $\gamma_{\min }$. The reason is that the slope of the spectrum is very sensitive to the number of scatterings, which increases with optical depth.

Because of the significant flow velocity in the jet, the upscattered photons escape preferentially in the forward direction. Thus we expect the results to also depend on the angle $\theta$ of observation with respect to the jet axis. The hardest spectra are found at small observation angles, as these are the photons that have suffered the most energetic scatterings. The cutoff energy increases as the observation angle decreases, but stabilizes at intermediate angles. However, once the angle range is fixed, the parameters follow a similar trend with optical depth and Lorentz gamma factor, irrespective of the range considered.

We also computed the time lag of hard photons in the energy range 7-40 keV with respect to softer photons in the range $2-7 \mathrm{keV}$. The time-lag spectrum, that is the time lag as a function 


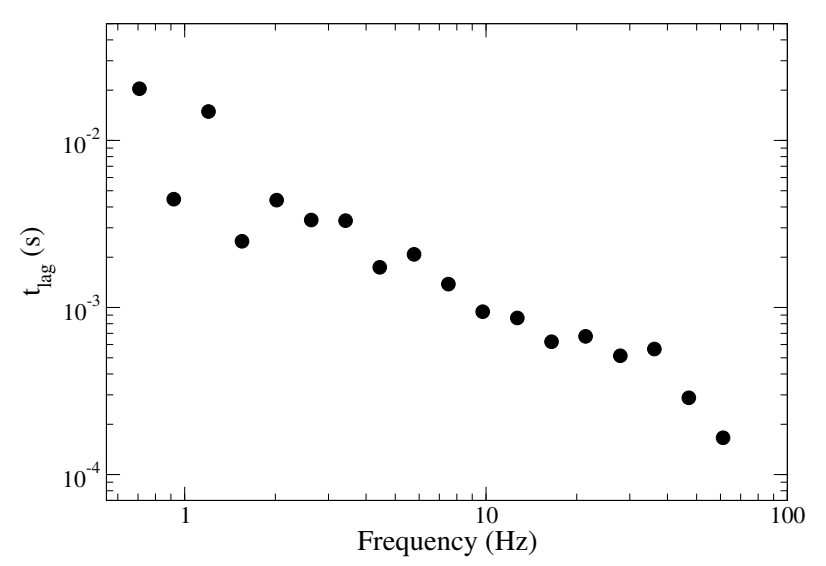

Fig. 3. Time-lag spectrum that results from the model with $\tau_{\|}=1$ and $\gamma_{\min }=3.34$. All the remaining parameters of the model were fixed at the reference values (see Sect. 2).

of Fourier frequency, is shown in Fig. 3. The spectra follow a power-law dependence on Fourier frequency $t_{\text {lag }} \sim v^{-\beta}$, with $\beta=1.0 \pm 0.1$. This slope is somewhat steeper than the average slope measured in black-hole binaries, $t_{\text {lag }} \sim v^{-\alpha}$ with $\alpha=0.7 \pm 0.1$ (Nowak et al. 1999; Pottschmidt et al. 2003; Cassatella et al. 2012), but agrees with the results reported by Olive \& Barret (2001). We have not found a significant dependence of the shape of the lag spectrum with either the optical depth or $\gamma_{\min }$.

\section{Discussion}

We ran Monte Carlo simulations of the Compton upscattering of soft photons in an outflow moving at relativistic velocity (representing a radio jet) and computed the emerging X-ray spectrum. The input parameters of the model have been selected so that the results match what is seen in bright NSXB. Assuming a blackbody input source of photons with $k T=2.5 \mathrm{keV}$, our model reproduces very well the observed $1-300 \mathrm{keV}$ X-ray spectrum in the harder states, a power law continuum with a photon index $\Gamma$ in the range 1.8-3 without evidence for a cutoff up to 200-300 keV.

The main difficulty of the models that seek to reproduce the high-energy X-ray spectral continuum in NSXB lies in explaining the absence of a cutoff, because the spectrum resulting from Comptonization is expected to roll over at a certain energy (an effect known as Compton recoil), when the energy of the photons approaches that of the electrons. Various models have been put forward to explain the absence of a cutoff in the hard tails: bulk-motion Comptonization (Bradshaw et al. 2003; Paizis et al. 2006; Farinelli et al. 2007, 2008), Comptonization by a hybridthermal-non thermal corona (Gierliński et al. 1999; Özel et al. 2000; Zdziarski et al. 2001; Farinelli et al. 2005; Revnivtsev et al. 2014), or synchrotron emission from the electrons of a jet (Markoff et al. 2005). Because of the limited sensitivity of current detectors at energies above $\sim 100 \mathrm{keV}$, statistically significant detections are given up to $\sim 200 \mathrm{keV}$, typically. The spectral continuum could in principle still roll over at a certain energy provided that this cutoff energy is larger than the limit energy of the detectors ${ }^{2}$. Recently, Revnivtsev et al. (2014) reported the

\footnotetext{
2 From the point of view of the energetics, if the slope of the power law is $\Gamma \leq 2$, then the spectrum must show a rollover at a certain energy to prevent a diverging luminosity.
}

highest lower limit on the cutoff energy that has been detected so far: $E_{\mathrm{c}}>330 \mathrm{keV}$, at a $2 \sigma$ significance level.

This relatively high lower limit of the cutoff energy was used by Revnivtsev et al. (2014) to rule out the bulk-motion Comptonization model. The reason for this is that the cutoff energy in thermal and bulk-motion Comptonization depends on the temperature and the velocity of the Comptonizing electrons, respectively. For realistic values of $k T_{\mathrm{e}}<50 \mathrm{keV}$ and assuming free-fall velocity $v_{\mathrm{ff}} \approx 0.5 c$ onto a neutron star of canonical mass $\left(1.4 M_{\odot}\right)$ and radius $\left(10^{6} \mathrm{~cm}\right)$, the cutoff energy is expected to be below $200 \mathrm{keV}$. Moreover, the radiation pressure expected to result from the emission of the neutron-star surface may reduce the infall velocity to less than $0.2 c$, decreasing the bulk-motion Comptonization effect (Farinelli et al. 2008).

Observations collected over the past decade show that there exists a clear connection between the X-ray spectral states and the radio emission in NSXB. Similarly to BHXB, the NSXB are radio-loud in the hard state, usually displaying a flat spectrum indicative of a relativistic jet. The radio emission is quenched when the source enters the soft state in most (but not all) cases (Penninx et al. 1988; Hasinger et al. 1990; Hjellming et al. 1990; Oosterbroek et al. 1994; Migliari \& Fender 2006; Migliari et al. 2007; Miller-Jones et al. 2010).

Motivated by the disputed origin of the hard X-ray tail in NSXB and by the clear relationship between the presence of this hard component and radio emission from the source in the form of a jet, we have investigated whether the observed X-ray spectrum of NSXB can be reproduced by our jet model. Our goal has been to reproduce the observed spectrum above $\sim 20 \mathrm{keV}$, namely, a power-law distribution with a photon index in the range 1.8-3 and the absence of a high-energy cutoff up to an observed energy of $300 \mathrm{keV}$. In Sect. 3, we demonstrated that such a range of photon indices can be obtained with an optical depth varying in the range $0.1 \leq \tau_{\|} \leq 1.5$ (Table 1 ). The lack of a cutoff in the observed spectra translates into cutoff energies above $200 \mathrm{keV}$ in our models. These energies are obtained when the electrons move with $\gamma_{\min } \gtrsim 3.2\left(v_{\perp} \gtrsim 0.52 c\right.$, for $v_{\|}=0.8 c$ ). We note that, in principle, and contrary to the bulk-motion Comptonization model, there is no restriction on the velocity components of the electrons in the jet, provided that the parallel component is significantly larger than the perpendicular component. Therefore, although we selected models that give cutoff energies above but not too far from $300 \mathrm{keV}$, we could increase our limit on $E_{\mathrm{c}}$ simply by increasing $\gamma_{\text {min }}$ (see Fig 2). We also note that such high $\gamma_{\min }$ is possible, since Fomalont et al. (2001), who studied the evolution of the radio emission in Sco $\mathrm{X}-1$, found that $v>0.95 c$.

\subsection{Comparison with black-hole binaries}

There are two major differences between the jets in NSXB and BHXB: i) the radio luminosities of neutron-star jets are typically 30 times lower than those of black-hole jets at comparable X-ray luminosities (Fender \& Kuulkers 2001; Migliari et al. 2003); and ii) radio emission is not completely quenched in the soft state of some NSXB (Migliari et al. 2004), as opposed to their non-detection in BHXB, when they are in the same state.

Both of these differences have been addressed successfully in Kylafis et al. (2012), where the formation and the destruction of jets is explained using the Cosmic Battery (Contopoulos \& Kazanas 1998; Contopoulos et al. 2006; Christodoulou et al. 2008).

That the radio luminosity is significantly lower in neutronstar jets than in black-hole ones can be understood as 
follows: while the radio luminosity scales as $\dot{m} M$ for both types of compact object (here, $\dot{m}$ is the mass accretion rate in units of the Eddington value and $M$ is the mass of the compact object), the X-ray luminosity due to the ADAF-like inner accretion flow scales as $\dot{m} M$ for neutron stars and as $\dot{m}^{2} M$ for black holes (Narayan et al. 1997; Migliari \& Fender 2006; Abramowicz \& Fragile 2013). Thus, for comparable X-ray luminosities, the radio luminosity of neutron-star jets is $\sim \dot{m}$ times smaller than that of black-hole ones.

The radio emission is quenched in the soft state of BHXB, because the Cosmic Battery works very inefficiently when the accretion flow is in the form of a Shakura-Sunyayev type (Shakura \& Sunyaev 1973). On the other hand, in low-mass NSXB with weak magnetic field $\left(B \sim 10^{8}-10^{9} \mathrm{G}\right)$, the Cosmic Battery can work moderately efficiently due to the "spreading layer" (Inogamov \& Sunyaev 2010) that forms on the surface of the neutron star, provided that there is a significant difference between the spin frequency of the neutron star and the Keplerian frequency of the disk.

The findings from our calculations are consistent with the characteristics of neutron-star and black-hole jets discussed above. We have found that in NSXB the required optical depths are in the range $0.1 \leq \tau_{\|} \leq 1.5$, while in BHXB they are in the range $1 \leq \tau_{\|} \leq 10$ (Reig et al. 2003; Kylafis et al. 2008; Reig \& Kylafis 2015). Because the optical depth is proportional to density and so is the radio emission, the lower optical depth in NSXB is consistent with the observational fact that the radio emission of the jet in NSXB is significantly smaller than that in BHXB.

To explain the fact that BHXB exhibit a high-energy cutoff in the X-ray spectrum while NSXB do not, we were forced to assume that $\gamma_{\min }$ in the jets of NSXB is larger than in BHXB. In particular, we found from our calculations that for BHXB $\gamma_{\min } \lesssim$ 2.4 (Reig \& Kylafis 2015), while for NSXB $\gamma_{\min } \gtrsim 3$. It will be interesting to see if this is confirmed by observations.

In a recent paper (Koljonen et al. 2015), an important anticorrelation was found between the break frequency $v_{b}$ in the jet spectrum (spectral energy distribution) and the power-law in$\operatorname{dex} \Gamma$ of the hard X-ray spectrum for BHXB and AGN. If the $\mathrm{X}$-ray spectrum is produced in the jet by inverse Compton scattering (Reig et al. 2003; Giannios et al. 2004; Giannios 2005; Kylafis et al. 2008; Reig \& Kylafis 2015), then this anticorrelation can be understood at least qualitatively. As the jet weakens, $\tau_{\|}$decreases, and as a result $\Gamma$ increases (Reig \& Kylafis 2015) and $v_{b}$ decreases. If our model is correct, that is to say if the hard X-ray spectrum is produced in the jet by inverse Comptonization, then we predict that NSXB as a class will exhibit lower values of $v_{b}$ than BHXB.

In our model, time or phase lags result from the random walk of the photons in the scattering medium. Hard photons scatter on average more times than softer ones before escaping the Comptonizing medium (the jet), hence they are delayed with respect to the softer ones. While the amplitude of the lags depends on the optical depth and dimensions of the jet (Giannios et al. 2004; Kylafis et al. 2008), the slope of the time-lag spectrum appears to be rather insensitive to those parameters or the Lorentz factor. In practice, the slope is determined for given energy ranges of the soft and hard photons and calculated for a given frequency range. Although most of our models produce slopes of $\sim 1$, smaller indices, similar to those of BHXB, could be obtained by different combinations of frequency and energy ranges. Given the lack of publications reporting Fourier time-lag spectra of NSXB, we cannot conclusively establish whether the different slopes that our calculations suggest corresponds to a distinguishing feature between NSXB and BHXB.

\section{Conclusion}

We ran Monte Carlo simulations of the Compton upscattering of soft photons in an outflow moving at relativistic velocity (representing a radio jet) and computed the emerging X-ray spectrum of bright NSXB. Our Monte Carlo simulations reproduce the observed spectral features: a power-law energy distribution at energies above $20 \mathrm{keV}$ with photon index in the range 1.8-3 and the absence of a high energy cutoff up to $\sim 300 \mathrm{keV}$. We demonstrate that the hard tail detected in these systems can be explained by Comptonization in the jet. The observed connection between the hard X-ray tail and the radio emission in low-mass X-ray binaries with neutron star companions supports this scenario. We explain the differences in the spectral and timing parameters between BHXB and NSXB by assuming that the electron population in the jets of NSXB have higher velocities and lower densities than in BHXB.

Acknowledgements. This research has been supported in part by the "RoboPol" project, which is implemented under the "ARISTEIA" action of the "Operational Program Education and Lifelong Learning" and is co-funded by the European Social Fund (ESF) and National Resources.

\section{References}

Abramowicz, M. A., \& Fragile, P. C. 2013, Liv. Rev. Rel., 16, 1

Band, D. L., \& Grindlay, J. E. 1986, ApJ, 311, 595

Barret, D. 2004, in Plasmas in the Laboratory and in the Universe: New Insights and New Challenges, eds. G. Bertin, D. Farina, \& R. Pozzoli, AIP Conf. Ser., 703, 238

Barret, D., Olive, J. F., Boirin, L., et al. 2000, ApJ, 533, 329

Bradshaw, C. F., Geldzahler, B. J., \& Fomalont, E. B. 2003, ApJ, 592, 486

Cassatella, P., Uttley, P., Wilms, J., \& Poutanen, J. 2012, MNRAS, 422, 2407

Christodoulou, D. M., Contopoulos, I., \& Kazanas, D. 2008, ApJ, 674, 388

Church, M. J., Gibiec, A., Bałucińska-Church, M., \& Jackson, N. K. 2012, A\&A, 546, A 35

Contopoulos, I., \& Kazanas, D. 1998, ApJ, 508, 859

Contopoulos, I., Kazanas, D., \& Christodoulou, D. M. 2006, ApJ, 652, 1451

D'Aí, A., Życki, P., Di Salvo, T., et al. 2007, ApJ, 667, 411

D'Amico, F., Heindl, W. A., Rothschild, R. E., \& Gruber, D. E. 2001, ApJ, 547, L147

Di Salvo, T., Stella, L., Robba, N. R., et al. 2000, ApJ, 544, L119

Di Salvo, T., Robba, N. R., Iaria, R., et al. 2001, ApJ, 554, 49

Di Salvo, T., Farinelli, R., Burderi, L., et al. 2002, A\&A, 386, 535

Di Salvo, T., Iaria, R., Robba, N., \& Burderi, L. 2006, Chin. J. Astron. Astrophys. Suppl., 6, 183

Ding, G. Q., Zhang, S. N., Wang, N., Qu, J. L., \& Yan, S. P. 2011, AJ, 142, 34

Done, C., Gierliński, M., \& Kubota, A. 2007, A\&ARv, 15, 1

Esin, A. A., McClintock, J. E., \& Narayan, R. 1997, ApJ, 489, 865

Farinelli, R., Frontera, F., Zdziarski, A. A., et al. 2005, A\&A, 434, 25

Farinelli, R., Titarchuk, L., \& Frontera, F. 2007, ApJ, 662, 1167

Farinelli, R., Titarchuk, L., Paizis, A., \& Frontera, F. 2008, ApJ, 680, 602

Farinelli, R., Paizis, A., Landi, R., \& Titarchuk, L. 2009, A\&A, 498, 509

Fender, R. P., \& Kuulkers, E. 2001, MNRAS, 324, 923

Fender, R. P., Belloni, T. M., \& Gallo, E. 2004, MNRAS, 355, 1105

Fender, R. P., Homan, J., \& Belloni, T. M. 2009, MNRAS, 396, 1370

Fomalont, E. B., Geldzahler, B. J., \& Bradshaw, C. F. 2001, ApJ, 553, L27

Garain, S. K., Ghosh, H., \& Chakrabarti, S. K. 2012, ApJ, 758, 114

Georganopoulos, M., Aharonian, F. A., \& Kirk, J. G. 2002, A\&A, 388, L25

Ghosh, H., Garain, S. K., Giri, K., \& Chakrabarti, S. K. 2011, MNRAS, 416, 959

Giannios, D. 2005, A\&A, 437, 1007 (Paper III)

Giannios, D., Kylafis, N. D., \& Psaltis, D. 2004, A\&A, 425, 163 (Paper II)

Gierliński, M., Zdziarski, A. A., Poutanen, J., et al. 1999, MNRAS, 309, 496

Gilfanov, M., Revnivtsev, M., \& Molkov, S. 2003, A\&A, 410, 217

Hasinger, G., \& van der Klis, M. 1989, A\&A, 225, 79

Hasinger, G., van der Klis, M., Ebisawa, K., Dotani, T., \& Mitsuda, K. 1990, A\&A, 235, 131

Hjellming, R. M., Stewart, R. T., White, G. L., et al. 1990, ApJ, 365, 681 
Homan, J., Wijnands, R., Rupen, M. P., et al. 2004, A\&A, 418, 255

Iaria, R., Burderi, L., Di Salvo, T., La Barbera, A., \& Robba, N. R. 2001, ApJ, 547,412

Inogamov, N. A., \& Sunyaev, R. A. 2010, Astron. Lett., 36, 848

Koljonen, K. I. I., Russell, D. M., Fernández-Ontiveros, J. A., et al. 2015, ApJ, 814,139

Kylafis, N. D., Papadakis, I. E., Reig, P., Giannios, D., \& Pooley, G. G. 2008, A\&A, 489, 481 (Paper IV)

Kylafis, N. D., Contopoulos, I., Kazanas, D., \& Christodoulou, D. M. 2012, A\&A, 538, A5

Lin, D., Remillard, R. A., \& Homan, J. 2007, ApJ, 667, 1073

Maccarone, T. J., \& Coppi, P. S. 2003, MNRAS, 338, 189

Markoff, S., Nowak, M. A., \& Wilms, J. 2005, ApJ, 635, 1203

McClintock, J. E., \& Remillard, R. A. 2006, in Black hole binaries, eds. W. H. G. Lewin, \& M. van der Klis, 157

Migliari, S., \& Fender, R. P. 2006, MNRAS, 366, 79

Migliari, S., Fender, R. P., Rupen, M., et al. 2003, MNRAS, 342, L67

Migliari, S., Fender, R. P., Rupen, M., et al. 2004, MNRAS, 351, 186

Migliari, S., Miller-Jones, J. C. A., Fender, R. P., et al. 2007, ApJ, 671, 706

Miller-Jones, J. C. A., Sivakoff, G. R., Altamirano, D., et al. 2010, ApJ, 716, L109

Mitsuda, K., Inoue, H., Koyama, K., et al. 1984, PASJ, 36, 741

Muñoz-Darias, T., Fender, R. P., Motta, S. E., \& Belloni, T. M. 2014, MNRAS, 443,3270

Narayan, R., \& Yi, I. 1994, ApJ, 428, L13
Narayan, R., Garcia, M. R., \& McClintock, J. E. 1997, ApJ, 478, L79

Nowak, M. A., Vaughan, B. A., Wilms, J., Dove, J. B., \& Begelman, M. C. 1999, ApJ, 510, 874

Olive, J.-F., \& Barret, D. 2001, in X-ray Astronomy: Stellar Endpoints, AGN, and the Diffuse X-ray Background, AIP Conf. Proc., 599, 814

Oosterbroek, T., Lewin, W. H. G., van Paradijs, J., et al. 1994, A\&A, 281, 803

Özel, F., Psaltis, D., \& Narayan, R. 2000, ApJ, 541, 234

Paizis, A., Farinelli, R., Titarchuk, L., et al. 2006, A\&A, 459, 187

Penninx, W., Lewin, W. H. G., Zijlstra, A. A., Mitsuda, K., \& van Paradijs, J. 1988, Nature, 336, 146

Pottschmidt, K., Wilms, J., Nowak, M. A., et al. 2003, A\&A, 407, 1039

Poutanen, J., Krolik, J. H., \& Ryde, F. 1997, MNRAS, 292, L21

Pozdnyakov, L. A., Sobol, I. M., \& Syunyaev, R. A. 1983, Astrophys. Space Phys. Rev., 2, 189

Reig, P., \& Kylafis, N. 2015, A\&A, 584, A109

Reig, P., Kylafis, N. D., \& Giannios, D. 2003, A\&A, 403, L15 (Paper I)

Revnivtsev, M. G., Tsygankov, S. S., Churazov, E. M., \& Krivonos, R. A. 2014, MNRAS, 445, 1205

Shakura, N. I., \& Sunyaev, R. A. 1973, A\&A, 24, 337

van der Klis, M. 1994, ApJS, 92, 511

van der Klis, M. 2005, Ap\&SS, 300, 149

van der Klis, M. 2006, in Rapid X-ray Variability, eds. W. H. G. Lewin, \& M. van der Klis, 39

Zdziarski, A. A., Grove, J. E., Poutanen, J., Rao, A. R., \& Vadawale, S. V. 2001, ApJ, 554, L45 